\title{
Design of the transfer stretcher for old age bathing and simulation analysis on human body mechanics
}

\author{
LEI Lei ${ }^{1, a^{*}}$, XUAN Liang ${ }^{2, b}$, TAO Ying ${ }^{3, c}$ and GUAN Tianmin ${ }^{1, d}$ \\ ${ }^{1}$ School of Mechanical Engineering, Dalian Jiaotong University, Dalian 116028,China \\ ${ }^{2}$ School of Mechanical and Architectural Engineering, Jianghan University, Wuhan 430056, China \\ ${ }^{3}$ School of Graduate Dep., North China Institute of Aerospace Engineering, Langfang 065000, China

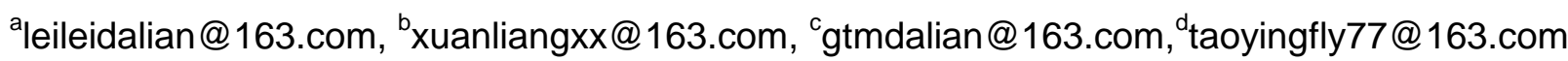

Keywords: Bath transfer stretcher; Lift back; Bending leg; Body mechanics simulation

Abstract. In this paper, in view of the stretcher transshipment transport stretcher part of car design modeling and simulation analysis, according to the design requirements for design transfer stretcher and with the back, legs Angle adjusting mechanism. AnyBody software is used to the human body back, leg movement simulation analysis, to select the reasonable of back, leg Angle adjusting range, provide the basis for transfer stretcher are comfortable design.

\section{Introduction}

In recent years, our country has been entering the aging society gradually. By 2025, China's aging population will reach more than 300 million. China will become the most serious country of aging population problem in developing countries. The elderly supplies industry has been rising with the emergence of an aging society. It has a huge market potential, not only bringing the good news to the elderly, but also becoming a new economic growth point ${ }^{[1]}$.

This article mainly aims at designing the elderly bathing transfer stretcher and making simulation analysis on it. Then design the transfer stretcher, its backrest, legs angle adjustment mechanism according to the design requirements. The AnyBody software is used to make simulation analysis on the human body's back, the movement of the legs bending. Select the rational backrest, the range of the legs angle adjustment to provide the basis for the comfort designing of transfer stretcher.

\section{The design requirements of the transfer stretcher}

The elderly lie on the transfer stretcher in the whole process of bathing. That is to say, the comfort of the transfer stretcher largely affect the quality of their bathing. So the transfer stretcher should not only be more designed from the perspective of the elderly's comfort.

The elderly have always been sitting or half-lying state in the process of bathing, so the weight makes different influence of the lumbar spine and lumbar muscle under different angel of the backrest. With good sitting posture, the pressure that the body bears assigns to each spinal segment reasonably. The intervertebral disc and tendons are under homogeneous static loading. When using a backrest with half-lying posture, part of the body weight is supported by the backrest. Meanwhile, the upper body has been upright state. The deformation of the spinal becomes small. Then ease the pressure on the spine tendons and intervertebral disc. When the elderly take in the shower, their head must be above the water. It determines the angle between the backrest and seat surface must be within a certain range $^{[2]}$.

\section{The design of the transfer stretcher and modeling}

Designing the transfer stretcher according to the adult body size of our country and using Pro/E to set up the entity model of the transfer stretcher. As shown in figure 1. 


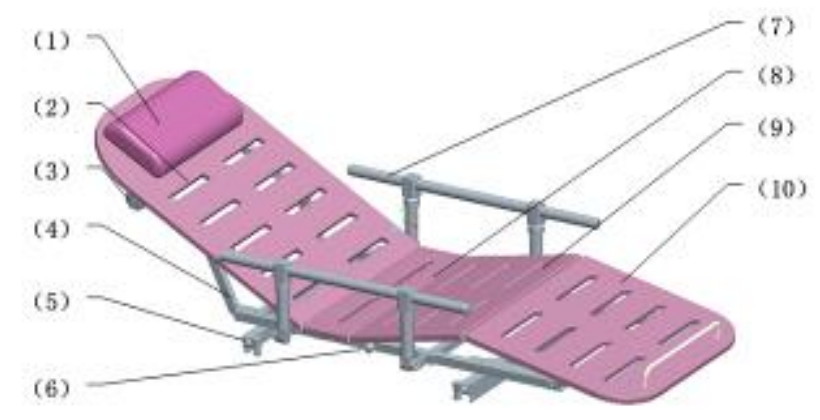

(1) pillow (2) back board (3) supporting wheel (4) ratchet wheel angle adjuster (5) transfer slipway

(6) self-lock spanner (7) armrest (8) buttocks board (9) thigh board (10) calf board Fig.1 Model of the transfer stretcher

The supporting board beyond the base frame is composed of back board, buttocks board, thigh board and calf board. Among them, the buttocks board is fixed by bolts. The back board, thigh board and calf board can realize the function of angle adjusting of lifting the back and bending the legs by using the angle adjusting mechanism. Every supporting board connects to each other by spring folders. There is the armrest device on the both sides of the stretcher. It can realize upside down. Specific technical parameters as shown in table 1 。

Table 1 technical parameters of the transfer stretcher

\begin{tabular}{cc}
\hline project & Technical parameters \\
\hline Dimension & $1800(\mathrm{~L}) \times 640(\mathrm{~W})$ \\
Quality & About $38 \mathrm{~kg}$ \\
& Frame: 304 Stainless \\
steel, \\
aluminun; \\
& Back board : ABS \\
plastic \\
Function & Rise back: $0^{\circ} \sim 60^{\circ} ;$ \\
& Bend legs: $0^{\circ} \sim 20^{\circ}$ \\
\hline
\end{tabular}

Mechanism design of the transfer stretcher's backrest.According to the design requirements, the transfer stretcher should have the function of rising the back to make the pressure distribute evenly on the back of the elderly who are paralyzed and reduce the pressure on shoulder when lie on their back.

In order to achieve that the transfer stretcher's angle of the back can be adjustable, selecting the angle adjustment device that the angle can be adjustable and installing the ratchet wheel angle adjuster between the back board and the base frame. One end fixes on the transfer stretcher's base frame, the other end installs rollers that can roll in the slipway of the back board. When the back board lifts up more than $60^{\circ}$, it can return to the horizontal position by the reset mechanism of the ratchet wheel angle adjuster. As shown in figure 2. 


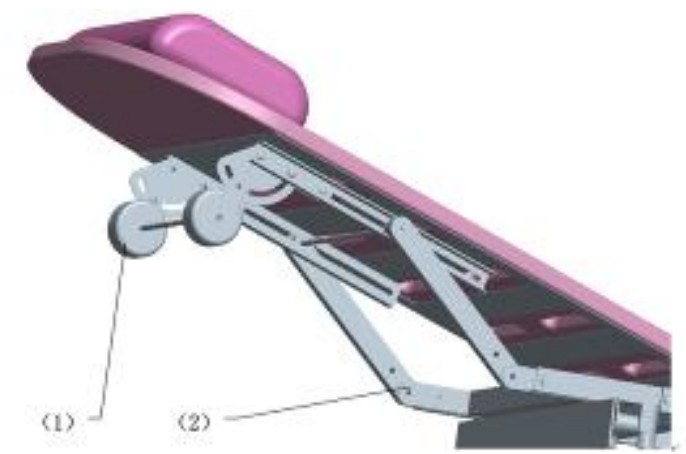

(1) supporting wheel (2) ratchet wheel angle adjuster

Fig. 2 Diagram of the backrest

There are some supporting wheels on the back of the backrest. After the transfer stretcher has been pushed to the bathtub, the bathtub lifts up and the inner wall of it will touch the supporting wheels to make the backrest rise up, that prevent the paralyzed old man from drowning because of low angle of the backrest. The supporting wheel also has the function of adjusting angle. So it's viable to adjust the angle of the backrest within a small range to satisfy different height of the paralyzed old man.

The design of the transfer stretcher's bending legs function.The adjustment of bending legs is another adjustment mechanism of the stretcher. When the paralyzed old men are taking in the shower, their knees bend in nature state because of the impact of water flow in the bathtub and the buoyancy of the legs. In order to increase the touching area between the paralyzed old man and the stretcher as much as possible and make the pressure distribute evenly. There must be the adjustment mechanism of bending legs. Reducing the pressure on the buttocks by increasing the touching area between the body and stretcher to make the muscles of the legs and joints appear relaxed posture.

The design of the adjustment mechanism of bending legs as shown in figure 3 . The thigh board connects to the calf board by using two spring folders. Two slipways are installed in the inside of the base frame of the stretcher symmetrically. There is a row of equidistance round hole on the inside slipway. The spring pulling pin that is installed on the outside slipway will get stuck in the round hole of the inside slipway when pulling the calf board. This design is like the front-back sliding adjustment mechanism of the car seat. It can achieve adjusting the angle of the thigh board between $0^{\circ}$ to $20^{\circ}$ by pulling the calf board.

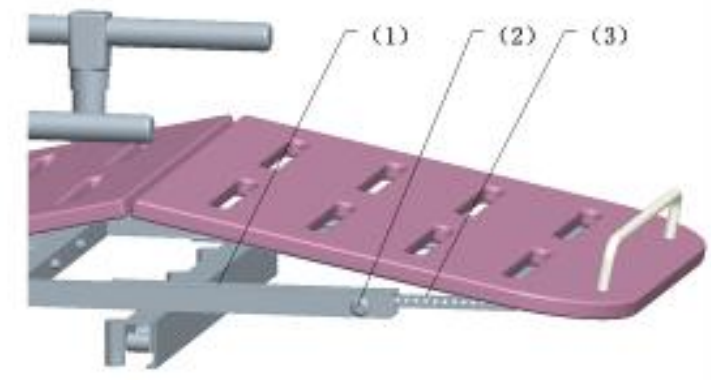

(1) outside slipway (2) spring pulling pin (3) inside slipway

Fig. 3 Diagram of the bending legs mechanism

\section{Set up the body model and simulation}

AnyBody human modeling and simulation system is a kind of modeling and simulation software about human movement simulation and analysis of muscle that specifically applied to ergonomics and biomechanics and so on fields. ${ }^{[4]}$

Modify the body model in AnyBody software. Skeletal muscle is a very complex mechanics executive mechanism. There are more than about 600 pieces of skeletal muscle in the human body 
which according to complex pattern determined by the central nervous system, boosting our bones to produce movement. So we are unable to establish a complete model of the human skeletal muscle by ourselves.

When simulating the lifting-back movement of human body, import the human body model file. Set the related parameters of height and weight to the value that based on the elderly of our country. Set the initial posture of the human model to prone position.

Modify the end time and step length of the whole human simulation model at the end of the program. Set the end time to 3 and the step length to 100. At last, because of considering the influence of gravity on the human body, add vertical downward acceleration of gravity 9.8 On the Y coordinate direction.

Simulation analysis of the lifting-back movement.First, after modifying the size of the human body model reasonably, set the initial simulation position to make the human body a lying posture. Import the model that is similar to the transfer stretcher to the human body simulation model to simulate the lifting-back movement of human body. Analysis the muscle movement about the lifting-back of human body according to the degree of muscle activity. The degree of muscle activity is an analytical data inherently of AnyBody software, which is a comprehensive date concluded by the external forces, passive force muscle and the changes of muscle strength caused by muscle contraction. So it's reasonable to choose the degree of muscle activity to be the measurement standard. Then do the simulating.
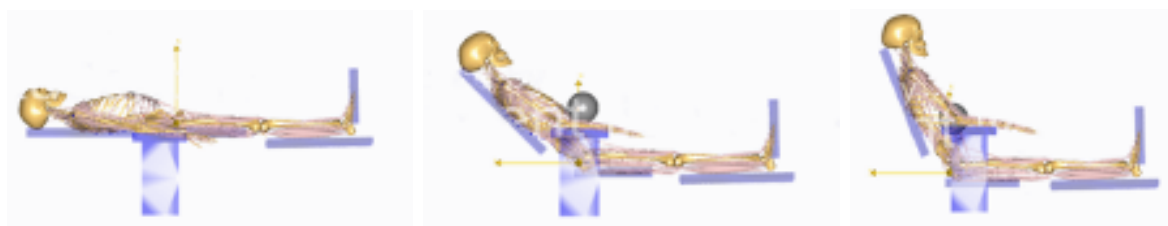

Fig. 4 The simulation of the rising-back movement of human body

It can be seen that the angle of the lifting-back changes $0^{\circ}$ to $60^{\circ}$ when the human body' s posture from lying state to half-lying state in the simulation window. As shown in figure 4. Open the date window after simulating. Click the button of the degree of the muscle activity. It will appear the time-varying graph of the degree of the muscle activity. As shown in figure 5.

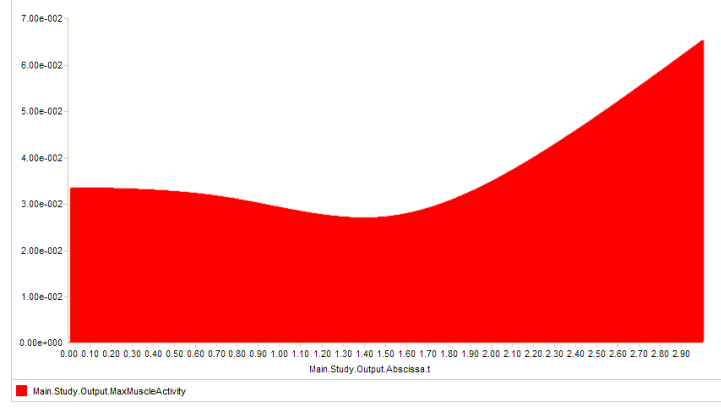

Fig. 5 The graph of the degree of upper limb muscle activity

The results showed, the degree of muscle activity will decrease slowly when the backrest is adjusted from lying position to half-lying position in the relaxing situation that the human body is only under the force of gravity. It increases dramatically when the position becomes the lowest.

It is concluded by collecting hundreds of muscle changes information of human upper limb that through mathematical analysis. This curve is a comprehensive simulation date, which is not only a analysis result of one muscle alone. So it's necessary to measure the degree of muscle activity of some representative muscle tissue.

Two muscles which mainly participated in are the back spinal muscle and the rectus abdominis. As shown in the red area of figure 6. The back spinal muscle changes initial shape of "long-thin" to the shape of "short-thick" gradually during the movement process of sitting position to lying position. Meanwhile the rectus abdominis changes initial shape of "short-thick" to the shape of "long-thin" gradually. So select the most representative muscle groups which are the back spinal muscle and the 
rectus abdominis to be the analysis of the reference of lifting-back movement after comprehensively analyzing the upper limb muscle groups.
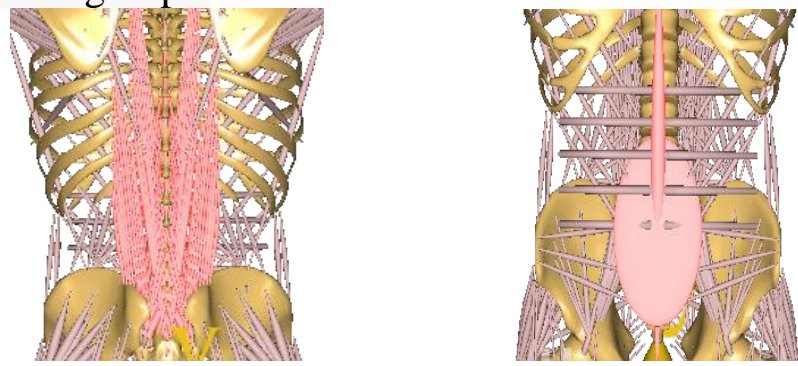

Fig. 6 the model of the back spinal muscle and the rectus abdominis in AnyBody

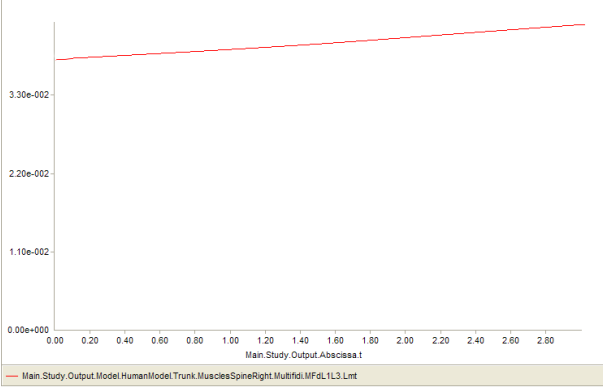

Fig. 7 The tensile curve of the back spinal muscle activity

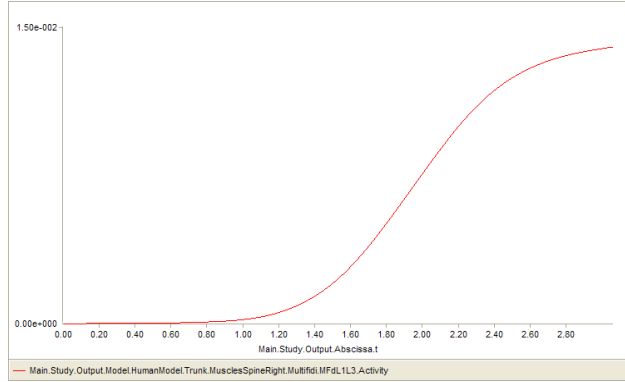

Fig. 8 the degree of back spinal muscle

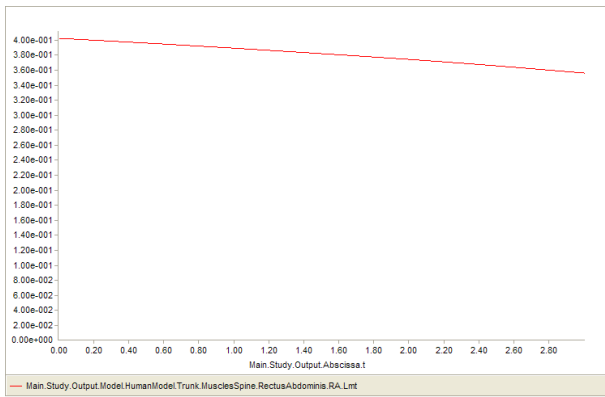

Fig. 9 the contraction curve of the rectus abdominis

It can be seen from the figure 7, the back spinal muscle will be pulled from $33 \mathrm{~mm}$ to $44 \mathrm{~mm}$ when the body adjusts the lying position to half-lying position. Stretching process is gentle.

The degree of the back spinal muscle activity has increased gradually during the lifting-back process from the figure 8 .

It can be seen that the rectus abdominis changes initial length of $49 \mathrm{~mm}$ to the length of $36 \mathrm{~mm}$ during the movement of the body changes the lying position to half-lying position. The length of curve of the rectus abdominis is a trend of slow decrease.

Based on the analysis of the above results, it can be seen that the body's minimum range of the degree of the muscle activity is about between $20^{\circ}$ to $40^{\circ}$ during the lifting-back process. It is just the range that the transfer stretcher could achieve.

Simulation analysis of the bending legs movement.Import the body sitting model to the body model. Then adjust the angle of the bending legs. Simulate the movement process of the body's legs from the bending legs state to straight legs state. As shown in figure 10. 


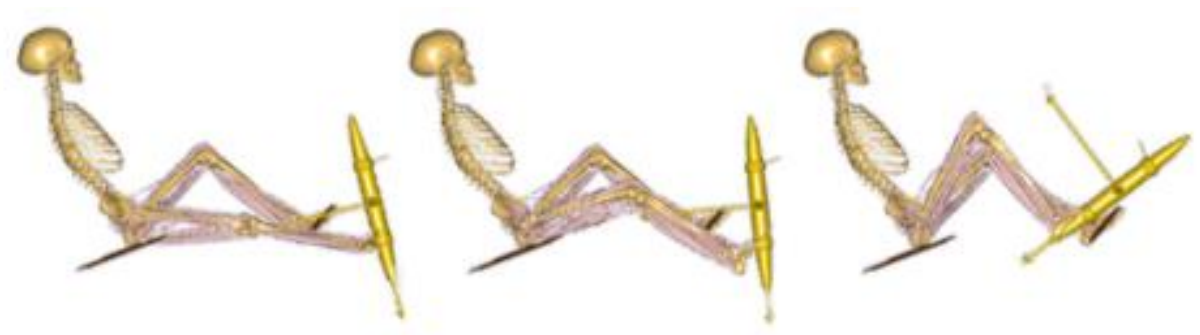

Fig.10 the simulation of the body bending legs

Analysis the degree of the body's legs muscle activity once again. Then get the changing curve of the degree of muscle activity as shown in figure 11. The degree of muscle activity will decrease suddenly during the legs from straight state to bending state when in the situation that the body is only under the force of gravity. Then it increases slowly.

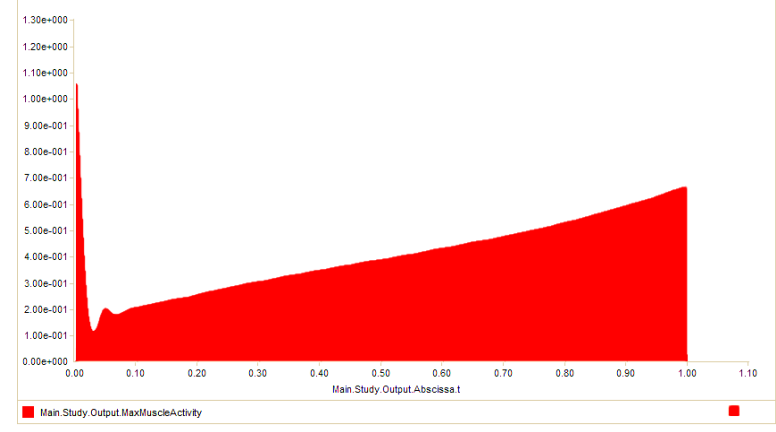

Fig. 11 the curve of the degree of lower limb muscle activity

From the above analysis results, the degree of muscle activity's minimum range is between about $0^{\circ}$ to $10^{\circ}$ during the process of the body bends legs. It is just the range that the bending legs adjuster of the transfer stretcher could achieve.

\section{Summary}

In this paper, the general architecture design has been accomplished according to the design requirements. And so do the new type of the backrest and the bending legs mechanism. By the Anybody software, set up the body model and simulate the movement of the body's lifting-back and bending legs. Then do the comprehensive analyzing on the degree of muscle activity during the movement process. Also analysis the stretch and the changes of the degree of muscle activity of the rectus abdominis and the back spinal muscle during the lifting-back movement .From the simulation data, determine the angle range of the backrest and bending legs which best meets the ergonomics. And then come to the conclusion that the angle adjustment of the backrest and the bending legs is reasonable.

\section{Acknowledgment}

This work was supported both by "Science and technology plan projects of Liaoning province" (No. 2013408005) and "Science and technology plan projects of Dalian, Liaoning province "(No. 2014A11GX043).

\section{References}

[1] Fang Tiancheng. Analysis of the development status and trends of Medical Devices Industry[J]. Financial viewpoint. 2012(11):157-158

[2] Liang Hongzhi. The design and development of the old man with horizontal shower stretcher[D].Master degree thesis of Dalian Jiaotong University.2014 
[3] Zhang Xiaoyu. Human factors engineering study on the bath environment of old people. Scientific and technological information [J]. 2010(1):359-364

[4] Cai Feng. Design and research of shoulder joint rehabilitation training robot [D]. Dalian: Master degree thesis of Dalian Jiaotong University, 2012.6

[5] Shan Lijun, Hu Zhong'an. Analysis of muscle strength of hip joint rehabilitation training based on AnyBody [J]. Journal of Dalian Jiaotong University, 2014

[6] Song Zhipeng. Research on human machine function analysis technology based on Radar Structure [D]. Xi'an Electronic and Science University, 2012

[7] Pan Dalei, Gao Feng. The relationship between body design and human body biomechanics of the lower limbs [J]. China Mechanical Engineering Conference, 2012.7.3 